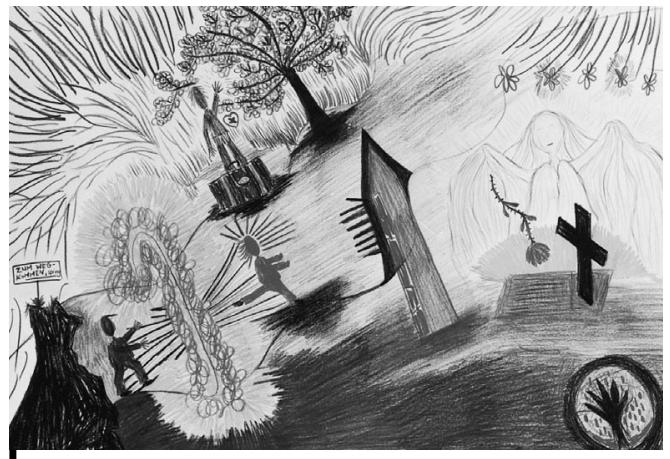

Erläuterungen zum Titelbild

(C) Copyright Manfred Fichter, Klinik Roseneck, Prien

Kurzanamnese und Wesentliches zum Verlauf

\section{Kommentar zur} Gestaltungstherapie

\section{Bildinterpretation}

Die 21-jährige Praktikantin lebt noch bei ihren Eltern. Nach dem unerwarteten Suizid ihres Freundes treten ausgeprägte depressive Verstimmungen mit Suizidphantasien, Hoffnungslosigkeit und innerer Leere auf. Zur Spannungsreduktion trinkt sie vermehrt Alkohol, raucht Cannabis und verletzt sich des Öfteren mit Rasierklingen. Ihre Struktur- und Ziellosigkeit führt zu massiven Konflikten mit den Eltern. Nach differentialdiagnostischer Abklärung ergibt sich eine Major Depression (einzelne Episode), eine posttraumatische Belastungsstörung sowie ein intermittierender Alkohol- und Drogenmissbrauch.

Die Patientin wird als mittlere von 3 Töchtern in einer Unternehmerfamilie geboren. Die Mutter beschreibt sie als beschützend und gutmütig, aber auch traurig und häufig unsicher, während der Vater als eher zurückgezogen, anspruchsvoll, zum einen schweigsam, andererseits aber auch jähzornig beschrieben wird. Als Kind leidet sie unter der ständigen Angst, dass sich die Eltern trennen könnten, was auch vorübergehend eintritt, als sie 15 Jahre alt ist. Im Alter von 12 Jahren stirbt ihre beste Freundin und deren gesamte Familie bei einem Autounfall. Sie trauert sehr intensiv um sie. In der 12. Klasse lernt sie ihren Freund kennen, zu dem sie eine sehr innige Beziehung entwickelt. Der Freund hat ein Drogenproblem, ist jedoch stets positiver Stimmung, bis er anfängt, unter Ängsten und Versagensgefühlen zu leiden, und sich 3 Monate darauf ohne jegliche Vorankündigung oder Abschiedsbrief durch Erhängen suizidiert.

Der Suizid des Freundes und die Bearbeitung der Angst auslösenden Flashbacks sind zentrale Themen zu Beginn der Therapie. Die schrittweise Konfrontation mit den inneren Bildern und Erinnerungen und den damit einher gehenden Gefühlen entlastet die Patientin. Die Häufigkeit der Flashbacks reduziert sich, der Spannungs- und Selbstverletzungsdruck lässt nach und ist nach etwa 4 Wochen verschwunden. Dysfunktionale Gedanken wie «ich hätte meinen Freund vor dem Suizid retten müssen» und «ich muss ihm ein Leben lang treu sein» werden kritisch hinterfragt und gehen in eine konstruktivere Sichtweise über. Es gelingt ihr schließlich, sich von der Idee, dem Freund in den Tod folgen zu müssen, zu distanzieren. Die familiäre Situation wird in der Gruppentherapie thematisiert und in Rollenspielen bearbeitet. Am Ende der Therapie berichtet sie, dass sie wieder neuen Lebensmut entwickelt und an innerer Freiheit gewonnen hat. Sie wirkt selbstsicher und zielstrebiger im Kontakt. Die Stimmungslage ist wesentlich verbessert und stabilisiert.

Bei der Bearbeitung der Flashbacks spielt die Gestaltungstherapie eine wichtige Rolle, da die Patientin hier erstmals bildlich darstellen kann, was ihr zuvor verbal nicht gelungen ist. Einige der Gestaltungen bilden die Grundlage für Einzeltherapiestunden, in denen die Erlebnisse nochmals intensiv nachbearbeitet werden können. Ihre Gefühlslage wird in ihren Bildern sehr deutlich: Einmal stellt sie sich z.B. auf dem Boden liegend dar, handlungsunfähig, eingesperrt hinter Gitterstäben. Daneben lodert ein kräftiges Feuer, das gleichzeitig Lebenskraft, Wut und Jenseitsstreben symbolisiert. Die Reichhaltigkeit des Dargestellten bietet neben dem Gewinn neuer Einsichten für die Patientin verschiedene Ansatzpunkte für verhaltenstherapeutische Maßnahmen, so z.B. das bewusste Herausgehen aus dem eigenen Gefängnis durch das Aufnehmen sozialer Kontakte.

In ihrem letzen Bild in der Gestaltungstherapie, das auf der Titelseite zu sehen ist, sieht man sie als dem Tode (schwarzer Fels links unten) entronnene Figur, die durch eine Phase der Geborgenheit im Elternhaus («rosa Watte»), in das sie nach dem Klinikaufenthalt vorübergehend zurück kehren wird, hindurch geht. Danach tritt sie neuen Aufgaben entgegen, die ambivalent besetzt sind: Anziehendes, Angenehmes (rosa Gebilde) und zu Bewältigendes, Unangenehmes (schwarze, abwehrende «Stacheln») vermischen sich. Das Grab des Freundes (rechts) wird von einem Engel bewacht - sie hat die Verantwortung für seinen Tod abgeben können. Die schwarze Hand, Symbol des Todes und der Angst davor (unten rechts), die in einem ersten Bild noch übermächtig nach ihr greift, ist zwar noch vorhanden, aber in einer festen Umkreisung gebannt. Die Patientin kann sich vorstellen, erst einmal auf Reisen zu gehen (Bild oben, Mitte). Die Nähe zum Lebensbaum zeigt, dass das Suchen neuer und eigener Wege eine wichtige, anstehende Selbsterfahrung ist.

Klinik Roseneck, Prien am Chiemsee Abteilung Gestaltungstherapie Text: Carl Leibl / Brit Wiethüchter / Angelika Neumann 